Afonso, A., Schmiedl, S., Becker, C., Tcherny-Lessenot, S., Primatesta, P., Plana, E., Souvereing P., Wang, Y., Korevaar, J.C., Hasford, J., Reynolds, R., Groot, M.C.H. de, Schlienger, R., Klungel, O., Rottenkolber, M. A methodological comparison of two European primary care databases and replication in a US claims database: inhaled long-acting beta-2-agonists and the risk of ive acute myocardial infarction. European Journal of Clinical Pharmacology: 2016, 72(9), 1105-11怕el

\begin{tabular}{l|l} 
Postprint $\quad 1.0$
\end{tabular}

Version

Journal website http://link.springer.com/article/10.1007\%2Fs00228-016-2071-8

Pubmed link https://www.ncbi.nlm.nih.gov/pubmed/27216032

DOI 10.1007/s00228-016-2071-8

This is a NIVEL certified Post Print, more info at http://www.nivel.eu

\title{
A methodological comparison of two European primary care databases and replication in a US claims database: inhaled long-acting beta-2- agonists and the risk of acute myocardial infarction
}

\author{
A. AFONSO ${ }^{1} \&$ S. SCHMIEDL ${ }^{2,3} \&$ C. BECKER ${ }^{4} \&$ S. TCHERNY-LESSENOT ${ }^{5} \&$ P. PRIMATESTA ${ }^{6}$ \\ \& E. PLANA ${ }^{7} \&$ P. SOUVEREIN ${ }^{1} \&$ Y. WANG ${ }^{5} \&$ J. C. KOREVAAR ${ }^{8} \&$ J. HASFORD ${ }^{9} \&$ R. \\ REYNOLDS ${ }^{10} \&$ M. C. H. DE GROOT ${ }^{1} \&$ R. SCHLIENGER ${ }^{6} \&$ O. KLUNGEL $^{1} \&$ M. \\ ROTTENKOLBER $^{9}$ \\ 1.Division Pharmacoepidemiology and Clinical Pharmacology, Utrecht Institute for \\ Pharmaceutical SciencesUtrecht UniversityUtrechtThe Netherlands \\ 2.Department of Clinical Pharmacology, School of Medicine, Faculty of \\ HealthWitten/Herdecke UniversityWittenGermany \\ 3.Philipp Klee-Institute for Clinical PharmacologyHELIOS Clinic \\ WuppertalWuppertalGermany \\ 4.Basel Pharmacoepidemiology Unit, Division of Clinical Pharmacy \& Epidemiology, \\ Department of Pharmaceutical SciencesUniversity of BaselBaselSwitzerland \\ 5.Global Pharmacovigilance and EpidemiologySanofiChilly-MazarinFrance \\ 6.Global EpidemiologyNovartis Pharma AGBaselSwitzerland \\ 7.Novartis Pharma AGBarcelonaSpain \\ 8.NIVEL, Netherlands Institute for Health Services ResearchUtrechtThe Netherlands \\ 9. Institute for Medical Information Sciences, Biometry, and EpidemiologyLudwig- \\ Maximilians-UniversitaetMunichGermany \\ 10.EpidemiologyPfizerNew YorkUSA
}

\begin{abstract}
Purpose Results from observational studies on inhaled long-acting beta-2agonists (LABA) and acute myocardial infarction (AMI) risk are conflicting, presumably due to variation in methodology. We aimed to evaluate the impact of applying a common study protocol on consistency of results in three databases.

Methods In the primary analysis, we included patients from two GP databases (Dutch-Mondriaan, UK - CPRD GOLD) with a diagnosis of asthma and/or COPD and at least one inhaled LABA or a "non-LABA inhaled bronchodilator medication" (short-acting beta-2-agonist or short-long-acting muscarinic antagonist) prescription between 2002 and 2009. A claims database (USAClinformatics) was used for replication. LABA use was divided into current, recent (first 91 days following the end of a treatment episode), and past use (after more than 91 days following the end of a treatment episode). Adjusted
\end{abstract}


Afonso, A., Schmiedl, S., Becker, C., Tcherny-Lessenot, S., Primatesta, P., Plana, E., Souverein P., Wang, Y., Korevaar, J.C., Hasford, J., Reynolds, R., Groot, M.C.H. de, Schlienger, R., Klungel, ) O., Rottenkolber, M. A methodological comparison of two European primary care databases 7 and replication in a US claims database: inhaled long-acting beta-2-agonists and the risk of ivel
acute myocardial infarction. European Journal of Clinical Pharmacology: 2016, 72(9), 1105-11估 hazard ratios (AMI-aHR) and $95 \%$ confidence intervals (95\% CI) were estimated using time-dependent multivariable Cox regression models stratified by recorded diagnoses (asthma, COPD, or both asthma and COPD).

Results For asthma or COPD patients, no statistically significant AMI-aHRs (age- and sex-adjusted) were found in the primary analysis. For patients with both diagnoses, a decreased AMI-aHR was found for current vs. recent LABA use in the CPRD GOLD (0.78; $95 \%$ CI 0.68-0.90) and in Mondriaan (0.55; $95 \%$ CI 0.28-1.08), too. The replication study yielded similar results. Adjusting for concomitant medication use and comorbidities, in addition to age and sex, had little impact on the results.

Conclusions By using a common protocol, we observed similar results in the primary analysis performed in two GP databases and in the replication study in a claims database. Regarding differences between databases, a common protocol facilitates interpreting results due to minimized methodological variations. However, results of multinational comparative observational studies might be affected by bias not fully addressed by a common protocol.

\section{INTRODUCTION}

Asthma and chronic obstructive pulmonary disease (COPD) are two of the most prevalent chronic airway diseases globally, and inhaled short- and long-acting beta2-adrenoceptor agonists (B2A) (SABA and LABA) are recommended as bronchodilator drugs for both indications by existing guidelines [1,2]. Due to betaadrenergic stimulation, cardiac events (e.g., tachyarrhythmias) are well-known adverse reactions for this drug group. In a meta-analysis of randomized, placebocontrolled trials (RCTs) in patients with obstructive airway disease, the risk for sinus tachycardia was significantly increased in B2A users compared to placebo (RR 3.06; $95 \%$ CI 1.7-5.5), whereas for major cardiovascular adverse events (including ventricular tachycardia, atrial fibrillation, syncope, congestive heart failure, acute myocardial infarction (AMI), cardiac arrest, and sudden death), the respective RR was 1.61 (95\% CI 0.76-3.42) not reaching statistical significance [3]. Since RCTs are usually not designed to detect differences in AMI risks between treatment groups, observational studies might be helpful to assess an association between B2A usage and AMI.

In a case-control study conducted in The Netherlands, no increased AMI risk was found for current B2A-agonist users (short- and long-acting combined) compared to never use (aOR 1.18, 95 \% CI 0.93-1.49) [4]. Similar results were observed in COPD patients with cardiac risk factors for fatal and nonfatal AMI for current SABA use compared to no use in the past year (adjusted rate ratio 1.17, $95 \% \mathrm{CI} 0.97-1.41$ ) [5]. On the other hand, an adjusted OR of 3.22 (95\% CI 1.63-6.35) for AMI was found in patients from the USA with a history of cardiovascular diseases receiving SABA in the 3 months prior to the AMI compared to nonusers which was more pronounced in new users (aOR 7.32, 95 \% CI 2.34-22.8) [6]. In Dutch patients with obstructive pulmonary diseases, an increased risk for sudden cardiac arrest was found for SABA (aOR 3.9, 95 \% CI 1.7-8.8) [7]. Heavy long-term users of SABA (at least 13 prescriptions in the year before) living in the UK had an increased AMI risk compared to users receiving less than three prescriptions (relative rate 1.6), 
Afonso, A., Schmiedl, S., Becker, C., Tcherny-Lessenot, S., Primatesta, P., Plana, E., Souvereing P., Wang, Y., Korevaar, J.C., Hasford, J., Reynolds, R., Groot, M.C.H. de, Schlienger, R., Klungel, O., Rottenkolber, M. A methodological comparison of two European primary care databases and replication in a US claims database: inhaled long-acting beta-2-agonists and the risk of jVel acute myocardial infarction. European Journal of Clinical Pharmacology: 2016, 72(9), 1105-11 V6 whereas for LABA, the respective value was 1.1 [8]. In patients from England receiving the LABA compound salmeterol, neither an increased risk for nonfatal cardiac failure (adjusted relative risk 1.10, 95 \% CI 0.63-1.91) nor for ischemic heart disease (adjusted relative risk 1.07, $95 \%$ CI 0.69-1.66) was found compared to patients receiving nedocromil [9]. To sum up, for SABA, some evidence was found showing an increased AMI risk in patients initiating treatment, in heavy users, and if cardiovascular comorbidities were present, whereas for LABA, only a few AMI risk estimates were reported.

Methodological reasons for differing AMI risk estimates are numerous. For example, pharmacoepidemiological (PE) studies may differ with regard to database characteristics (e.g., claims vs. GP database), inclusion criteria (age restrictions, comorbidities, co-medications), exposure (consideration of dosages), follow-up period, outcome (identification and classification of AMI) definition, and the confounders adjusted for in multivariate models (comorbidities, co-medications, disease severity) [8, 10-13]. Whereas Zhang et al. [8] defined AMI cases by using the General Practice Research Database (GPRD) and the national registry of hospital admissions in England, the analyses of Au et al. [11] were based on patients included in a multicenter, randomized clinical trial. By comparing the populations included in the respective analyses, a selection bias might be more prominent regarding the results of $\mathrm{Au}$ et al. [11]. Furthermore, intercountry differences regarding prescribing habits and the health-care system can influence $\mathrm{PE}$ study results $[12,13]$. By comparing seven health-care record databases in five European countries, Rottenkolber et al. [13] found relevant intercountry differences regarding period prevalence rates of LABA-containing prescriptions and regarding the extent of potential off-label use of LABA. In addition, the choice of statistical methods for the control of confounding may also have contributed to differing results [10] and protopathic bias might be of particular relevance. For example, in some patients, dyspnea caused by an unknown severe coronary heart disease (CHD) might symptomatically be treated with B2A. In such patients, risk estimates for B2Arelated AMI might be overestimated due to nonconsideration of CHD [14]. We aimed to evaluate the impact of applying a common, uniform study protocol on consistency of risk estimates of AMI associated with LABA comparing two European electronic GP databases. Furthermore, we assessed the impact of methodological choices (e.g., adjustment for different confounding variables, different indications) on study results. To assess generalizability of our findings, a replication study was conducted in a United States (US) health insurance claims database using the same protocol.

\section{METHODS}

\section{Study setting and data sources}

This cohort study was performed in the general practice databases, GOLD of the Clinical Practice Research Datalink (CPRD, formerly GPRD [15]) and the Mondriaan project (including the Netherlands Primary Care Research Database which is maintained by NIVEL, the Almere Health Care database (AHC), and the Julius Primary Care Network) [16-18]. Details of these databases are shown in Table 1 and e-Table 1 and have been described elsewhere [10,17, 19-21]. 
Afonso, A., Schmiedl, S., Becker, C., Tcherny-Lessenot, S., Primatesta, P., Plana, E., Souvereing P., Wang, Y., Korevaar, J.C., Hasford, J., Reynolds, R., Groot, M.C.H. de, Schlienger, R., Klungel, O., Rottenkolber, M. A methodological comparison of two European primary care databases and replication in a US claims database: inhaled long-acting beta-2-agonists and the risk of IVel $^{-}$ acute myocardial infarction. European Journal of Clinical Pharmacology: 2016, 72(9), 1105-11 v6

The CPRD GOLD contains data of more than five million active UK patients; diagnoses and drugs are coded using Read Codes [22] and Multilex Codes [23], respectively. For the CPRD GOLD database, the study protocol was approved by the Independent Scientific Advisory Committee (ISAC) of CPRD. Patient information is only available anonymized and deidentified in the database, and hence, no informed consent is needed from patients.

In the Mondriaan databases, information is available of about 1.4 million patients; diagnoses and drugs are coded using the International Classification in Primary Care (ICPC-1 [24]) and the Anatomical Therapeutic Chemical classification system (ATC [25]), respectively. For the Mondriaan databases, approval was obtained from the institutional review boards of each of the GP databases (Almere Health Care group, Netherlands Institute for Health Services Research, Julius General Practitioners Network). All data are anonymized and no explicit informed consent is needed according to Dutch law and regulations.

The replication study was performed in Clinformatics Data Mart (Clinformatics), a large US health insurance claims database containing data of more than 32.8 million patients, covering United HealthCare insurance plans including Medicaid [26, 27]. The International Classification of Diseases, Ninth Revision, Clinical Modification (ICD-9 CM [28]) was used for coding diagnoses and the National Drug Code Directory (NDC [29]) for coding drugs. For Clinformatics, no ethical approval is required. Since patient information is only available anonymized and deidentified in the database, no informed consent is needed from patients.

In this study, we maintained a blinding procedure until the final results were submitted to the coordinating center at Utrecht University. The detailed study protocol is available online [30].

\section{[TABLE 1]}

\section{Study population and cohorts}

All patients receiving at least one prescription of a SABA and/or LABA and/or inhaled short- and/or long-acting muscarinic antagonist (SAMA, LAMA) during the study period from 1 January 2002 to 31 December 2009 (in the AHC database from 1 January 2002 to 30 September 2008) were included in the study. Cohort entry date (index date) was defined as the date the first prescription of an inhaled SABA, LABA, SAMA, or LAMA during the study period was documented (following a 1year period of valid data documentation). All patients with a previous AMI or without a coded diagnosis of asthma or COPD were excluded. For the baseline characteristics, patients were assigned to the LABA group, the exposure of interest (e-Table 2), if they had at least one LABA prescription in the study period, irrespective of other prescriptions of inhaled asthma or COPD medications. Patients were considered as non-LABA patients if at least one SABA, SAMA, or LAMA but no LABA was prescribed during the study period. A 1-year period before the index date was considered for the assessment of baseline characteristics regarding comedications, whereas for comorbidities, all available observation time before the index date was used.

\section{Exposure definition, study outcome, and assessment of indication}

The duration of inhaled LABA use was determined by using prescription data to estimate continuous treatment periods. In the CPRD GOLD and in Clinformatics, the 
Afonso, A., Schmiedl, S., Becker, C., Tcherny-Lessenot, S., Primatesta, P., Plana, E., Souvereing P., Wang, Y., Korevaar, J.C., Hasford, J., Reynolds, R., Groot, M.C.H. de, Schlienger, R., Klungel, O., Rottenkolber, M. A methodological comparison of two European primary care databases 7 and replication in a US claims database: inhaled long-acting beta-2-agonists and the risk of ${ }_{1}$ el expected duration of each prescription was estimated using the prescribed quantity based on package sizes and the prescribed daily dose. In case of missing data (e.g., the daily dose or package size), the database-specific median treatment duration was used. In the Mondriaan database, a time period of 90 days (the typical period for prescribing medicines for chronic diseases in The Netherlands) was used as a surrogate since both parameters (daily dosage and package size) were often unknown.

The exposure time of inhaled LABA was divided into three different exposure time states (e-Fig. 1). For each patient, treatment episodes were created allowing no gaps between prescriptions within the same episode. End of a treatment episode was the calculated end date of the last prescription. The gap between treatment episodes was divided into recent and past use. Recent use was defined as the first 91 days following the end of a treatment episode. A user was considered to be a past user after 91 days had passed from the calculated end of a treatment episode and until a new treatment episode was initiated. Non-LABA use (SAMA and/or SABA and/or LAMA) was classified into two different states: from the date of the first prescription until the end of the treatment episode (current use) and after the end of the treatment period (no-current use, e-Fig. 2). The exposure state was handled as a timedependent variable in the Cox-proportional hazard models.

The first fatal or nonfatal AMI within the study period was the outcome of interest (e-Table 3). Patients were followed up until the earliest occurrence of one of the following events (end of follow-up): AMI, dropout, death, or end of study period. The indication for using LABA or SABA or SAMA or LAMA was defined using the date of the last respective prescription within the study period searching the entire database backwards for medical codes to classify the patients into the following mutually exclusive categories: "asthma" (diagnosis of asthma but no documented diagnosis of COPD), "COPD” (diagnosis of COPD but no documented diagnosis of asthma), and "asthma and COPD" (documented diagnosis of asthma and COPD) irrespective of any other (respiratory) diagnosis (e-Table 4). All analyses were performed in these subgroups defined by indication.

\section{Statistical analysis}

Incidence rates (AMI-IRs) were calculated as the number of AMI events divided by person-time. Crude incidence rate ratios (AMI-IRRs) and $95 \%$ confidence intervals (95\% CIs) were calculated by dividing the AMI-IR of current LABA use by the AMI-IR of the three successive comparison groups (recent LABA use; past LABA use; current non-LABA use) [31]. Time-dependent Cox regression models were used to estimate adjusted hazard ratios (AMI-aHRs). Model 1 was adjusted for age (timedependent) and sex only, whereas model 2 was adjusted for age (time-dependent), sex, comorbidities, and concomitant medications.

Relevant comorbidities and concomitant medication use (e-Tables 5-7, e-Figs. 1 and 2 ) were handled as time-varying covariates. These time-dependent confounders were assessed every 6 months and in the case of exposure changes. Once comorbidity was diagnosed during a 6-month assessment period, it was considered as "present disease" for the whole study period onwards. In contrast, for co-medications, a patient could switch between use and nonuse groups at each 6-month assessment or in the case of exposure changes. Within the sensitivity analyses (performed in CPRD only), the impact of different lengths of treatment periods and of additional 
Afonso, A., Schmiedl, S., Becker, C., Tcherny-Lessenot, S., Primatesta, P., Plana, E., Souvereing P., Wang, Y., Korevaar, J.C., Hasford, J., Reynolds, R., Groot, M.C.H. de, Schlienger, R., Klungel, O., Rottenkolber, M. A methodological comparison of two European primary care databases 7 and replication in a US claims database: inhaled long-acting beta-2-agonists and the risk of $\mathrm{j}_{\mathrm{V}} \mathrm{e}$
acute myocardial infarction. European Journal of Clinical Pharmacology: 2016, 72(9), 1105-119. stratifications of LABA and non-LABA use was examined. Details of the sensitivity analyses are shown in e-Fig. 3.

All statistical calculations were performed using SAS statistical software package, version 9.3 (SAS Institute Inc., Cary, NC, USA).

\section{RESULTS}

In the CPRD GOLD, almost two thirds of patients $(656,414$ out of $1,037,647$ people; $63.3 \%$ ) with at least one LABA and/or SABA and/or LAMA and/or SAMA prescription during the study period fulfilled the inclusion criteria (Fig. 1). Of those, 540,382 (82.3\%) had an asthma diagnosis only, 47,056 (7.2\%) a COPD diagnosis only, and 68,976 (10.5\%) both diagnoses. In Mondriaan, 36,188 of 116,420 (31.1\%) patients fulfilled the inclusion criteria. Most patients were excluded due to missing COPD or asthma indication (78,723 [67.7\%]). In comparison to the CPRD GOLD, the proportion of COPD patients included in Mondriaan was higher $(\mathrm{n}=7720$ [21.3\%]), lower for asthma patients $(n=24,956$ [69.0\%]), and similar for asthma and COPD patients $(\mathrm{n}=3512$ [9.7\%]). In Clinformatics, 319,873 $(58.8 \%)$ out of 543,584 patients were included. Most of the patients had an asthma diagnosis only (n $=232,323[72.6 \%]), 43,691(13.7 \%)$ a COPD diagnosis only, and 43,859 (13\%) had both diagnoses (Fig. 1).

\section{[FIGURE 1]}

The proportion of patients with LABA prescriptions in Mondriaan was higher in all three respiratory diagnosis groups when compared to the CPRD GOLD and Clinformatics (Fig. 1).

\section{Baseline characteristics}

In the LABA group, asthma patients were older and the proportion of females was higher compared to patients in the non-LABA group across all databases (Table 2, eTables 8 and 10). COPD patient characteristics varied by database; in Clinformatics and Mondriaan, COPD patients were younger and more likely to be female than patients in the CPRD GOLD. COPD patients had evidence of more comorbidities than asthma patients and were prescribed more co-medications (Table 2, e-Tables 810). Patients with both asthma and COPD recorded ranked in the middle between patients diagnosed with asthma or COPD regarding distribution and frequency of baseline characteristics (Table 2, e-Tables 8-10).

\section{[TABLE 2]}

\section{Crude incidence rates and crude incidence rate ratios for AMI}

Figure 2 shows the AMI-IRs stratified by indication, exposure, and periods of exposure. The number of periods is shown in e-Table 11. For asthma patients, similar AMI-IRs were found in all databases with the highest IRs occurring during current LABA use (CPRD GOLD 16.5; Mondriaan 16.7; Clinformatics 17.4 per 10,000 person-years (PY)). For COPD patients and patients diagnosed with both, asthma and COPD, AMI-IRs were much higher and less comparable between databases. In both strata and for all databases, the highest AMI-IRs were found during recent LABA use. AMI-IRRs are shown in e-Table 12. 
Afonso, A., Schmiedl, S., Becker, C., Tcherny-Lessenot, S., Primatesta, P., Plana, E., Souvereing P., Wang, Y., Korevaar, J.C., Hasford, J., Reynolds, R., Groot, M.C.H. de, Schlienger, R., Klungel, O., Rottenkolber, M. A methodological comparison of two European primary care databases 7 and replication in a US claims database: inhaled long-acting beta-2-agonists and the risk of ive acute myocardial infarction. European Journal of Clinical Pharmacology: 2016, 72(9), 1105-11 V6

\section{Multivariate Cox model-adjusted hazard ratios}

Figure 3 and e-Table 13 provide the AMI-aHRs for all strata and databases. For asthma patients, the AMI-aHRs using models 1 and 2 varied slightly around the threshold of 1 . Results for COPD patients were similar, with a nonsignificant decreased risk of AMI during current $\mathrm{LABA}$ use when compared to recent LABA use (CPRD GOLD 0.90, 95 \% CI 0.72-1.14; Mondriaan 0.68, $95 \%$ CI 0.40-1.15) and significantly lower than 1.00 in Clinformatics (aHR 0.62, $95 \%$ CI 0.49-0.78, eTable 13, model 1). For patients diagnosed with both, asthma and COPD, a decreased risk of AMI was found for the comparison of current vs. recent LABA use, significant in the CPRD GOLD $(0.78,95 \%$ CI 0.68-0.90) and in Clinformatics $(0.71,95 \%$ CI $0.58-0.86)$, where as the comparison of current LABA use vs. current non-LABA use yielded significant results in Clinformatics only $(0.76,95 \% \mathrm{CI} 0.68-$ 0.85 , model 1). Additional adjustment for comorbidities and co-medications (model 2) did not change the results significantly (Fig. 3, e-Table 13).

\section{[FIGURE 2]}

\section{Sensitivity analyses}

Due to the low number of AMI events in the Mondriaan database, sensitivity analyses were only performed in the CPRD GOLD. In the replication study, no sensitivity analysis was planned. Changing the definition of recent and past LABA use and no-current non-LABA use from 91 to 30 days (e-Fig. 3) did not alter the study findings markedly. When stratifying LABA exposure according to concomitant usage of inhaled corticosteroids (e-Fig. 3), no significant changes were found in comparison to the primary analysis. In contrast, by stratifying non-LABA exposure according to SABA, SAMA, and LAMA (e-Fig. 3), in COPD patients, a statistically significant AMI-aHR was found for current LABA use vs. current SABA use (model $1-1.23$ (95 \% CI 1.03-1.47), model 2-1.25 (95\% CI 1.04-1.50)). For patients with asthma or with asthma and COPD, no significant changes compared to the main analysis were found (data not shown).

\section{Discussion}

By applying a common study protocol, we found similar risk estimates for AMI in patients receiving LABA across two European GP databases (primary analysis) and a US claims database (replication study). For most comparisons, the adjusted HRs were around 1 in all three databases. For some comparisons, significant results were found (e.g., current LABA use vs. recent LABA in patients diagnosed with both, asthma and COPD, in the CPRD GOLD and in the Clinformatics database). In Mondriaan, similar but nonsignificant results were observed. Since this study was primarily conducted as methodological exercise to compare results between different (types) of databases, a detailed medical discussion might be expendable.

\section{Impact of methodological choices}

In this study, additional adjustment for concomitant medication use and comorbidities (model 2) had only a minor impact on the results in all three databases. This might be related to a similar prevalence of risk factors (comorbidities, comedications) in LABA and non-LABA users (COPD patients and patients diagnosed with both, asthma and COPD). The observed prevalence differences of comorbidities 
Afonso, A., Schmiedl, S., Becker, C., Tcherny-Lessenot, S., Primatesta, P., Plana, E., Souvereing P., Wang, Y., Korevaar, J.C., Hasford, J., Reynolds, R., Groot, M.C.H. de, Schlienger, R., Klungel, O., Rottenkolber, M. A methodological comparison of two European primary care databases and replication in a US claims database: inhaled long-acting beta-2-agonists and the risk of $j_{\text {V }}$ e acute myocardial infarction. European Journal of Clinical Pharmacology: 2016, 72(9), 1105-11此 and co-medications between LABA and non-LABA patients with asthma may primarily reflect differences in age.

\section{[FIGURE 3]}

When the reference group was changed from past LABA use to recent LABA use, a decreased HR of current LABA use associated with AMI was observed in Clinformatics for COPD patients. This might be due to the fact that recent users have actually stopped the medication potentially for reasons that are related to the outcome of interest and therefore not necessarily a cause of the medication. From a medical as well as from a methodological point of view, an indicationspecific analysis discriminating patients diagnosed with asthma, COPD, or asthma and COPD seems reasonable. For example, in COPD patients, the highest prevalence rates of coronary heart disease - a main risk factor of AMI - were found. Hence, calculating indication-specific risk estimates is of outstanding importance, whereas overall analyses mixing different indications could mask drug effects. In addition, confounding may operate differently in subgroups with different indications.

\section{Potential sources of variation}

While Mondriaan and the CPRD GOLD are regional and national GP databases, respectively, Clinformatics is a claims database with a shorter average time of follow-up compared to both GP databases and only captures information on those encounters which generate a claim. Furthermore, coding systems of diseases and drugs differ between the three databases. For example, ICPC coding is a more general coding system, nonspecific or detailed compared to READ or ICD. Therefore, a comprehensive and careful mapping of codes was conducted to minimize those differences. However, some mismatches due to, e.g., a differing level of specificity cannot be excluded.

In addition, current use periods were defined in different ways. Whereas in Clinformatics, the exact treatment duration was calculated in all patients, default values for unknown pack size and for DDDs were used in 10 and $55 \%$ of prescriptions documented in the CPRD GOLD. In the Mondriaan database, an estimate of 90 days was used for all patients taking into account that in The Netherlands, drug prescriptions for chronic conditions usually cover a 3-month period. In addition, the mean treatment duration per prescription calculated in CPRD was 82 days (data not shown).

Published prevalence rates for asthma and COPD differ between The Netherlands, the UK, and the USA [32-34]. Differing proportions of patients diagnosed with asthma or COPD found in our study might reflect regional differences in prevalence, diagnostic practices, and health-care systems $[32,35]$. Nevertheless, the proportion of patients documented with COPD and asthma in terms of overlapping diseases [36] was similar between the three databases. Taking into account all these potential sources of variation, using a common protocol helped to conduct comparable database studies as shown by similar results found in the two initial cohort studies (CPRD GOLD, Mondriaan) and the replication study (Clinformatics).

\section{Study strengths and limitations}

Our study has several strengths. First, all analyses were initially conducted using a common protocol applied to two European GP databases allowing to minimize methodological differences [10]. In addition, findings were replicated using the same 
Afonso, A., Schmiedl, S., Becker, C., Tcherny-Lessenot, S., Primatesta, P., Plana, E., Souvereing P., Wang, Y., Korevaar, J.C., Hasford, J., Reynolds, R., Groot, M.C.H. de, Schlienger, R., Klungel, O., Rottenkolber, M. A methodological comparison of two European primary care databases and replication in a US claims database: inhaled long-acting beta-2-agonists and the risk of jVel acute myocardial infarction. European Journal of Clinical Pharmacology: 2016, 72(9), 1105-11 V6 protocol in a US health insurance claims database. All statistical analyses in the European databases and the US database were conducted independently and blinded. There are several study limitations worth mentioning. First, we could not include the severity of COPD/asthma and lifestyle factors as confounders due to the lack of relevant clinical and lifestyle information across all three databases. However, no relevant impact of considering such confounders on LABA-associated AMI risk estimates was found in other studies suggesting confounding by, e.g., lifestyle factors as already captured to a large extent by routinely collected confounders [37].

Similarly, for another drug-AE pair (benzodiazepines and hip/femur fractures) examined within the PROTECT project, consideration of additional lifestyle factors (e.g., alcohol, smoking) as part of a sensitivity analysis had no relevant impact on risk estimates in CPRD GOLD database [38]. However, we did include exposure information on several drugs prescribed for exacerbations (e.g., oral corticosteroids) as confounders in the sensitivity analysis, acting as a proxy for disease severity. In these analyses, adjustment of comorbidities and co-medications had little impact on the results suggesting that confounding may not have been a major issue in this study. Second, since no accurate information regarding the actual patient medication taking behavior or doses received was available across all databases, we could not consider dose-related aspects in our analysis. Hence, there is some uncertainty in terms of exposure, in particular for reliever medications used on an "as needed" basis such as SABA. Third, misclassification of asthma and COPD [39] might be present in all databases. Since validation of diagnoses was not the focus of this study, we did rely on documented diagnoses only. However, even for claims databases frequently lacking documented lung function parameters, a sufficient validity of respiratory diagnoses was reported [40-42]. Regarding a lower prevalence of cardiovascular diseases in non-COPD patients $[43,44]$, misclassifying these subjects as COPD patients will inflate the number of COPD patients having a decreased AMI risk. Taking into account a limited number of cardiovascular confounders considered in our analysis, AMI risk estimates will be influenced by misclassification of patients. Fourth, we did not distinguish incident from nonincident LABA users. Hence, an increased AMI risk - which has been reported in particular after initiating SABA and LABA treatment $[4,8]$ - might have been masked in our study. In addition, a distortion of the results due to the healthy adherer or the selection bias cannot be ruled out, given that prevalent users were included in our study. Furthermore, protopathic bias leading to a falsely increased LABA risk estimate due to LABA initiation in patients with dyspnea due to unknown/not documented cardiac disorders cannot be excluded. Fifth, a predefined common study protocol including a fixed set of selected confounders in multivariate models was used, regardless of their impact in each database which is not common practice for a bespoke pharmacoepidemiology comparative safety evaluation study. Nevertheless, this was cautiously addressed in our study and has been discussed in an earlier publication [10]. Sixth, the indication was defined retrospectively limiting application of our findings to a new population. Nevertheless, the retrospective approach was chosen for several reasons: Regarding the linkage between prescription and diagnosis, there were relevant differences between the databases. Taking into account the chronic character of both diseases and a delayed diagnosing of asthma or COPD commonly seen in clinical practice $[45,46]$, a retrospective approach might be meaningful. In addition, there is a particular uncertainty in diagnosing asthma COPD overlap syndrome (ACOS) [47]. 
Afonso, A., Schmiedl, S., Becker, C., Tcherny-Lessenot, S., Primatesta, P., Plana, E., Souvereing P., Wang, Y., Korevaar, J.C., Hasford, J., Reynolds, R., Groot, M.C.H. de, Schlienger, R., Klungel, O., Rottenkolber, M. A methodological comparison of two European primary care databases 7 and replication in a US claims database: inhaled long-acting beta-2-agonists and the risk of ${ }_{1} V_{6} \mathrm{el}$

To avoid bias in ACOS patients diagnosed first with asthma and afterwards with COPD (or vice versa), a retrospective assessment might be useful to identify ACOS patients having more severe clinical courses and comorbidities potentially influencing our study results $[47,48]$. Finally, we could not exclude differences in defining study outcome (AMI) between the countries (UK, The Netherlands, USA). As shown by Kontos et al. [49], a renewed AMI definition including troponin as a diagnostic marker led to a substantial increase in AMI cases. Nevertheless, since this definition was a consensus statement of the European Society of Cardiology and the American College of Cardiology published in 2000 [50], some standardization between the countries might have been reached for the study period and the databases included in our analyses. Despite the fact that no direct validation of AMI codes was conducted in our study, positive predictive values of 85 and $93 \%$ were reported in prior validation studies for the CPRD [51, 52]. Nevertheless, AMI-related analyses (at least in administrative databases) might have some shortcomings [53]. Furthermore, we abstained from linking death registries or autopsy data due to the primary methodological character of our study potentially limiting the external validity of our results.

To sum up, our study was conducted in two European GP databases and replicated in a US claims database, whereas former studies estimating AMI risks in LABA (or SABA) users were conducted in only one specific database [4-9]. We have shown in our study that applying a common study protocol to different databases is feasible, even if analyses are conducted in GP databases and in claims databases. Between the databases, risk estimates did only slightly differ underlining the robustness of our analysis. However, differences in coding of risk factors may limit the comparability of results. Furthermore, it should be taken into account that this study was primarily methodological in nature limiting clinical implications.

\section{CONCLUSION}

In conclusion, by using a common protocol in two European databases and replication in a US claims database, we found similar risk estimates for AMI in patients receiving LABA. From a methodological point of view, we confirmed usage of a common protocol as a feasible tool for conducting intercountry comparisons while minimizing methodological variation of studies. Databases reflect different health-care delivery and recording systems, but the interpretation of results can be improved since methods were harmonized via the common protocol.

\section{Acknowledgments}

The research leading to these results was conducted as part of the PROTECT consortium (Pharmacoepidemiological Research on Outcomes of Therapeutics by a European ConsorTium, www.imi-protect.eu) which is a public-private partnership coordinated by the European Medicines Agency.

The authors thank the excellent collaboration of physicians in the participating countries, whose contribution in recording their professional practice with high quality standards enables the availability of databases used in this research.

The paper is on behalf of the members of work-package 2 (WP2) and work-package 6 (WP6) of PROTECT. 
Afonso, A., Schmiedl, S., Becker, C., Tcherny-Lessenot, S., Primatesta, P., Plana, E., Souvereing P., Wang, Y., Korevaar, J.C., Hasford, J., Reynolds, R., Groot, M.C.H. de, Schlienger, R., Klungel, O., Rottenkolber, M. A methodological comparison of two European primary care databases 7 and replication in a US claims database: inhaled long-acting beta-2-agonists and the risk of $\mathrm{j}_{\mathrm{V}} \mathrm{e}$

\section{Compliance with ethical standards}

Conflict of interest AA, PS, JK, JH, and MR have no conflicts of interest; CB reports that her department at the University of Basel received payment from Novartis Pharma AG, Basel, Switzerland, during the conduct of the study for statistical analyses; MdG reports grants from Top Institute Pharma (NL) www.tipharma.com; SS reports personal fees from Rottapharm Madaus (Cologne, Germany) and travel costs for an investigator meeting reimbursed by Bayer HealthCare AG (Leverkusen, Germany), the division of Pharmacoepidemiology \& Clinical Pharmacology employing O.H. Klungel received Top Institute Pharma Grant T6.101 Mondriaan unrestricted grant for pharmacoepidemiological research. STL, PP, EP, YW, RR, and RS belong to EFPIA (European Federation of Pharmaceutical Industries and Association) member companies in the IMI JU and costs related to their part in the research were carried by the respective company as in-kind contribution under the IMI JU scheme. EP was previously employed at Novartis when this work was carried out. EP is publishing as a Novartis former employee. Funding The PROTECT project has received support from the Innovative Medicine Initiative Joint Undertaking (IMI JU; www.imi.europa.eu) under Grant Agreement no. 115004, resources of which are composed of financial contribution from the European Union's Seventh Framework Programme (FP7/2007-2013) and EFPIA (European Federation of Pharmaceutical Industries and Association) companies' inkind contribution. In the context of the IMI JU, the Department of Pharmacoepidemiology, Utrecht University, also received a direct financial contribution from Pfizer. The views expressed in this article are those of the authors only and not of their respective institution or company.

\section{REFERENCES}

1.

Global Initiative for Asthma (GINA) (2014) Global strategy for asthma management and prevention (revised 2014). [cited 2015 Dec 15]. Available from: 2. http://www.ginasthma.org/local/uploads/files/GINA_Report_2014_Aug12.pdf

Global Initiative for Chronic Obstructive Lung disease (GOLD) (2014) Global strategy for the diagnosis, management and prevention of COPD. [cited 2015 Dec 15]. Available from: http://www.goldcopd.org

3.

Salpeter SR, Ormiston TM, Salpeter EE (2004) Cardiovascular effects of beta-agonists in patients with asthma and COPD: a meta-analysis. Chest 125(6):2309_ 2321CrossRefPubMedGoogle Scholar

4.

de Vries F, Pouwels S, Bracke M, Lammers JW, Klungel O, Leufkens H, van Staa T (2008) Use of beta2 agonists and risk of acute myocardial infarction in patients with hypertension. Br J Clin Pharmacol 65(4):580-586. doi:10.1111/j.1365-2125.2007.03077.x CrossRefPubMedPubMedCentralGoogle Scholar

5.

Suissa S, Assimes T, Ernst P (2003) Inhaled short acting beta agonist use in COPD and the risk of acute myocardial infarction. Thorax 58(1):4346CrossRefPubMedPubMedCentralGoogle Scholar

6.

Au DH, Lemaitre RN, Curtis JR, Smith NL, Psaty BM (2000) The risk of myocardial infarction associated with inhaled beta-adrenoceptor agonists. Am J Respir Crit Care Med 161(3 Pt 1):827-830CrossRefPubMedGoogle Scholar 7. 
Afonso, A., Schmiedl, S., Becker, C., Tcherny-Lessenot, S., Primatesta, P., Plana, E., Souvereing P., Wang, Y., Korevaar, J.C., Hasford, J., Reynolds, R., Groot, M.C.H. de, Schlienger, R., Klungel, ) O., Rottenkolber, M. A methodological comparison of two European primary care databases 7 and replication in a US claims database: inhaled long-acting beta-2-agonists and the risk of $\mathrm{j}_{\mathrm{V}} \mathrm{e}$ Warnier MJ, Blom MT, Bardai A, Berdowksi J, Souverein PC, Hoes AW, Rutten FH, de Boer A, Koster RW, De Bruin ML, Tan HL (2013) Increased risk of sudden cardiac arrest in obstructive pulmonary disease: a case-control study. PLoS One 8(6):e65638. 8. doi:10.1371/journal.pone.0065638 CrossRefPubMedPubMedCentralGoogle Scholar

Zhang B, de Vries F, Setakis E, van Staa TP (2009) The pattern of risk of myocardial infarction in patients taking asthma medication: a study with the General Practice Research Database. J Hypertens 27(7):1485-1492. doi:10.1097/HJH.0b013e32832af68d CrossRefPubMedGoogle Scholar

9.

Martin RM, Dunn NR, Freemantle SN, Mann RD (1998) Risk of non-fatal cardiac failure and ischaemic heart disease with long acting beta 2 agonists. Thorax 53(7):558562CrossRefPubMedPubMedCentralGoogle Scholar 10.

Abbing-Karahagopian V, Kurz X, de Vries F, van Staa TP, Alvarez Y, Hesse U, Hasford J, Dijk L, de Abajo FJ, Weil JG, Grimaldi-Bensouda L, Egberts AC, Reynolds RF, Klungel OH (2014) Bridging differences in outcomes of pharmacoepidemiological studies: design and first results of the PROTECT project. Curr Clin Pharmacol 9(2):130 138CrossRefPubMedPubMedCentralGoogle Scholar 11.

Au DH, Curtis JR, Every NR, McDonell MB, Fihn SD (2002) Association between inhaled beta-agonists and the risk of unstable angina and myocardial infarction. Chest 121(3):846851CrossRefPubMedGoogle Scholar 12.

Bianchi M, Clavenna A, Bonati M (2010) Inter-country variations in anti-asthmatic drug prescriptions for children. Systematic review of studies published during the 2000-2009 period. Eur J Clin Pharmacol 66(9):929-936. doi:10.1007/s00228-010-0845-y CrossRefPubMedGoogle Scholar

13.

Rottenkolber M, Voogd E, van Dijk L, Primatesta P, Becker C, Schlienger R, de Groot MC, Alvarez Y, Durand J, Slattery J, Afonso A, Requena G, Gil M, Alvarez A, Hesse U, Gerlach $\mathrm{R}$, Hasford J, Fischer R, Klungel OH, Schmiedl S (2015) Time trends of period prevalence rates of patients with inhaled long-acting beta-2-agonists-containing prescriptions: a European comparative database study. PLoS One 10(2):e0117628. doi:10.1371/journal.pone.0117628 CrossRefPubMedPubMedCentralGoogle Scholar 14.

Feinstein AR, Horwitz RI (1978) A critique of the statistical evidence associating estrogens with endometrial cancer. Cancer Res 38(11 Pt 2):4001-4005PubMedGoogle Scholar 15.

Williams T, van Staa T, Puri S, Eaton S (2012) Recent advances in the utility and use of the General Practice Research Database as an example of a UK Primary Care Data resource. Ther Adv Drug Saf 3(2):89-99CrossRefPubMedPubMedCentralGoogle Scholar 16.

Clinical J Primary Care Research Network. In: ed. [cited 2015 Dec 15]. Available from: http://www.juliusclinical.com/global-presence\#cat10 17.

Stirbu-Wagner I, Dorsman SA, Visscher S, Davids R, J.V. G, Abrahamse H, van Althuis T, Jansen B, Schlief A, W. T, Walk C, Wentink E, Wennekes L, Braspenning J, Korevaar JC (2010) Landelijk Infomatienetwerk Huisartsenzorg. Feiten en cijfers over huisartsenzorg in Nederland. Utrecht/Nijmegen: NIVEL/IQ 18.

Top Institute Pharma TN. In: ed. [cited 2015 Dec 15]. Available from: http://www.tipharma.com/projects/efficiency-analysis-drug-discovery-process/themondriaan-project.html 19.

Herrett E, Thomas SL, Schoonen WM, Smeeth L, Hall AJ (2010) Validation and validity of diagnoses in the General Practice Research Database: a systematic review. Br J Clin 
Afonso, A., Schmiedl, S., Becker, C., Tcherny-Lessenot, S., Primatesta, P., Plana, E., Souverein P., Wang, Y., Korevaar, J.C., Hasford, J., Reynolds, R., Groot, M.C.H. de, Schlienger, R., Klungel, ) O., Rottenkolber, M. A methodological comparison of two European primary care databases 7 and replication in a US claims database: inhaled long-acting beta-2-agonists and the risk of $\mathrm{j}$ V el

Pharmacol 69(1):4-14. doi:10.1111/j.1365-2125.2009.03537.x

CrossRefPubMedPubMedCentralGoogle Scholar

20.

Jick SS, Kaye JA, Vasilakis-Scaramozza C, Garcia Rodriguez LA, Ruigomez A, Meier CR, Schlienger RG, Black C, Jick H (2003) Validity of the general practice research database. Pharmacotherapy 23(5):686-689CrossRefPubMedGoogle Scholar

21.

Lawrenson R, Williams T, Farmer R (1999) Clinical information for research; the use of general practice databases. J Public Health Med 21(3):299-304CrossRefPubMedGoogle Scholar

22.

NHS NHS Connecting for Health Read Codes. [cited 2015 Dec 15]. Available from: http://www.connectingforhealth.nhs.uk/systemsandservices/data/uktc/readcodes 23

MULTILEX. [cited 2015 Dec 15]. Available from: www.fdbhealth.co.uk/solutions/multilex/ 24.

The Classification Committee of WONCA (1998) ICPC-2, international classification of primary care. Oxford University Press, OxfordGoogle Scholar

25.

WHO (1992) Anatomical therapeutic chemical (ATC) classification index. WHO Collaborating Centre for Drug Statistics Methodology, GenevaGoogle Scholar 26.

Clinformatics Data Mart overview. [cited 2015 Dec 15]. Available from:

http://www.optuminsight.com/life-sciences/solutions/value-strategy/marketinganalytics/clinformatics-data-mart/overview

27.

Stergachis A, Saunders KW, Davis RL, Kimmel SE, Schinnar R, Chan KA, Shatin D, Rawson NSB, Hennessy S, Downey W, Stang M, Beck P, Osei W, Leufkens HG, Macdonald TM, Gelfand JM (2006) Examples of automated databases. Wiley, ChichesterCrossRefGoogle Scholar

28.

Statistics NCfH (2002) Classification of diseases and injuries. Ninth revision 29.

FDA National Drug Code Directory Data Files. [cited 2015 Dec 15]. Available from: http://www.accessdata.fda.gov/scripts/cder/ndc/default.cfm 30.

ENCePP (2014) European Network of Centres for Pharmacoepidemiology and Pharmacovigilance. [cited 2015 Dec 15]. Available from: http://www.encepp.eu/ 31.

Rothman KJ, Greenland S (2008) Modern epidemiology. Lippincott Williams \& Wilkins, PhiladelphiaGoogle Scholar

32.

Masoli M, Fabian D, Holt S, Beasley R, Global Initiative for Asthma P (2004) The global burden of asthma: executive summary of the GINA Dissemination Committee report. Allergy 59(5):469-478. doi:10.1111/j.1398-9995.2004.00526.x] CrossRefPubMedGoogle Scholar

33.

Afonso AS, Verhamme KM, Sturkenboom MC, Brusselle GG (2011) COPD in the general population: prevalence, incidence and survival. Respir Med 105(12):1872-1884. doi:10.1016/j.rmed.2011.06.012 CrossRefPubMedGoogle Scholar

34.

Soriano JB, Maier WC, Egger P, Visick G, Thakrar B, Sykes J, Pride NB (2000) Recent trends in physician diagnosed COPD in women and men in the UK. Thorax 55(9):789794CrossRefPubMedPubMedCentralGoogle Scholar 35.

Buist AS, McBurnie MA, Vollmer WM, Gillespie S, Burney P, Mannino DM, Menezes AM, Sullivan SD, Lee TA, Weiss KB, Jensen RL, Marks GB, Gulsvik A, NizankowskaMogilnicka $E$ (2007) International variation in the prevalence of COPD (the BOLD Study): a 
Afonso, A., Schmiedl, S., Becker, C., Tcherny-Lessenot, S., Primatesta, P., Plana, E., Souverein P., Wang, Y., Korevaar, J.C., Hasford, J., Reynolds, R., Groot, M.C.H. de, Schlienger, R., Klungel, O., Rottenkolber, M. A methodological comparison of two European primary care databases and replication in a US claims database: inhaled long-acting beta-2-agonists and the risk of jV $_{6} \mathrm{el}$ population-based prevalence study. Lancet 370(9589):741-750. doi:10.1016/S01406736(07)61377-4 CrossRefPubMedGoogle Scholar

36.

Gibson PG, Simpson JL (2009) The overlap syndrome of asthma and COPD: what are its features and how important is it? Thorax 64(8):728-735. doi:10.1136/thx.2008.108027 CrossRefPubMedGoogle Scholar

37.

Groenwold RHH, de Groot MCH, Ramamoorthy D, Souverein P, Klungel OH (2016) Unmeasured confounding in pharmacoepidemiology. Ann Epidemiol 26(1):85-86. doi:10.1016/j.annepidem.2015.10.007

38.

Requena G, Huerta C, Gardarsdottir H, Logie J, González-González R, AbbingKarahagopian V, Miret M, Schneider C, Souverein PC, Webb D, Afonso A, Boudiaf N, Martin E, Oliva B, Alvarez A, de Groot $\mathrm{MCH}$, Bate A, Johansson S, Schlienger R, Reynolds $\mathrm{R}$, Klungel $\mathrm{OH}$, de Abajo FJ (2015) Hip/femur fractures associated with the use of benzodiazepines (anxiolytics, hypnotics and related drugs): a methodological approach to assess consistencies across databases from the PROTECT-EU Project.

Pharmacoepidemiol Drug Saf 25(Supplement S1):66-78PubMedGoogle Scholar 39

Tinkelman DG, Price DB, Nordyke RJ, Halbert RJ (2006) Misdiagnosis of COPD and asthma in primary care patients 40 years of age and over. J Asthma 43(1):75-80. doi:10.1080/02770900500448738 CrossRefPubMedGoogle Scholar

40.

Lacasse Y, Montori VM, Lanthier C, Maltis F (2005) The validity of diagnosing chronic obstructive pulmonary disease from a large administrative database. Can Respir J 12(5):251-256CrossRefPubMedGoogle Scholar

41.

Macaulay D, Sun SX, Sorg RA, Yan SY, De G, Wu EQ, Simonelli PF (2013) Development and validation of a claims-based prediction model for COPD severity. Respir Med 107(10):1568-1577. doi:10.1016/j.rmed.2013.05.012 CrossRefPubMedGoogle Scholar 42.

Twiggs JE, Fifield J, Apter AJ, Jackson EA, Cushman RA (2002) Stratifying medical and pharmaceutical administrative claims as a method to identify pediatric asthma patients in a Medicaid managed care organization. J Clin Epidemiol 55(9):938944CrossRefPubMedGoogle Scholar 43.

Cazzola M, Bettoncelli G, Sessa E, Cricelli C, Biscione G (2010) Prevalence of comorbidities in patients with chronic obstructive pulmonary disease. Respiration 80(2):112-119. doi:10.1159/000281880 CrossRefPubMedGoogle Scholar 44.

Schnell K, Weiss CO, Lee T, Krishnan JA, Leff B, Wolff JL, Boyd C (2012) The prevalence of clinically-relevant comorbid conditions in patients with physician-diagnosed COPD: a crosssectional study using data from NHANES 1999-2008. BMC Pulm Med 12:26. doi:10.1186/1471-2466-12-26 CrossRefPubMedPubMedCentralGoogle Scholar 45.

Jagana R, Bartter T, Joshi M (2015) Delay in diagnosis of chronic obstructive pulmonary disease: reasons and solutions. Curr Opin Pulm Med 21(2):121-126. doi:10.1097/MCP.0000000000000133 CrossRefPubMedGoogle Scholar 46.

Molis WE, Bagniewski S, Weaver AL, Jacobson RM, Juhn YJ (2008) Timeliness of diagnosis of asthma in children and its predictors. Allergy 63(11):1529-1535. doi:10.1111/j.13989995.2008.01749.x CrossRefPubMedGoogle Scholar

47.

Papaiwannou A, Zarogoulidis P, Porpodis K, Spyratos D, Kioumis I, Pitsiou G, Pataka A, Tsakiridis K, Arikas S, Mpakas A, Tsiouda T, Katsikogiannis N, Kougioumtzi I, Machairiotis N, Siminelakis S, Kolettas A, Kessis G, Beleveslis T, Zarogoulidis K (2014) Asthma-chronic obstructive pulmonary disease overlap syndrome (ACOS): current literature review. J 
Afonso, A., Schmiedl, S., Becker, C., Tcherny-Lessenot, S., Primatesta, P., Plana, E., Souvereing P., Wang, Y., Korevaar, J.C., Hasford, J., Reynolds, R., Groot, M.C.H. de, Schlienger, R., Klungel, O., Rottenkolber, M. A methodological comparison of two European primary care databases 7 and replication in a US claims database: inhaled long-acting beta-2-agonists and the risk of $\mathrm{j}_{\mathrm{V}} \mathrm{e}$ Thorac Dis 6(Suppl 1):S146-S151. doi:10.3978/j.issn.2072-1439.2014.03.04

PubMedPubMedCentralGoogle Scholar

48

Hardin M, Silverman EK, Barr RG, Hansel NN, Schroeder JD, Make BJ, Crapo JD, Hersh CP (2011) The clinical features of the overlap between COPD and asthma. Respir Res 12:127. doi:10.1186/1465-9921-12-127 CrossRefPubMedPubMedCentralGoogle Scholar 49

Kontos MC, Fritz LM, Anderson FP, Tatum JL, Ornato JP, Jesse RL (2003) Impact of the troponin standard on the prevalence of acute myocardial infarction. Am Heart $\mathrm{J}$ 146(3):446-452. doi:10.1016/S0002-8703(03)00245-X CrossRefPubMedGoogle Scholar 50

Alpert JS, Thygesen K, Antman E, Bassand JP (2000) Myocardial infarction redefined-a consensus document of The Joint European Society of Cardiology/American College of Cardiology Committee for the redefinition of myocardial infarction. J Am Coll Cardiol 36(3):959-969CrossRefPubMedGoogle Scholar 51.

Hammad TA, McAdams MA, Feight A, Iyasu S, Dal Pan GJ (2008) Determining the predictive value of Read/OXMIS codes to identify incident acute myocardial infarction in the General Practice Research Database. Pharmacoepidemiol Drug Saf 17(12):1197-1201. doi:10.1002/pds.1672 CrossRefPubMedGoogle Scholar

52.

van Staa TP, Abenhaim L (1994) The quality of information recorded on a UK database of primary care records: a study of hospitalizations due to hypoglycemia and other conditions. Pharmacoepidemiol Drug Saf 3:15-21CrossRefGoogle Scholar

53.

McCormick N, Lacaille D, Bhole V, Avina-Zubieta JA (2014) Validity of myocardial infarction diagnoses in administrative databases: a systematic review. PLoS One 9(3):e92286. doi:10.1371/journal.pone.0092286 CrossRefPubMedPubMedCentralGoogle Scholar 
Afonso, A., Schmiedl, S., Becker, C., Tcherny-Lessenot, S., Primatesta, P., Plana, E., Souvereing P., Wang, Y., Korevaar, J.C., Hasford, J., Reynolds, R., Groot, M.C.H. de, Schlienger, R., Klungel, O., Rottenkolber, M. A methodological comparison of two European primary care databases 7

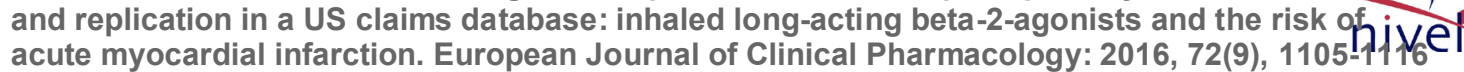

TABLES AND FIGURES 
Afonso, A., Schmiedl, S., Becker, C., Tcherny-Lessenot, S., Primatesta, P., Plana, E., Souvereing P., Wang, Y., Korevaar, J.C., Hasford, J., Reynolds, R., Groot, M.C.H. de, Schlienger, R., Klungel, O., Rottenkolber, M. A methodological comparison of two European primary care databases 7

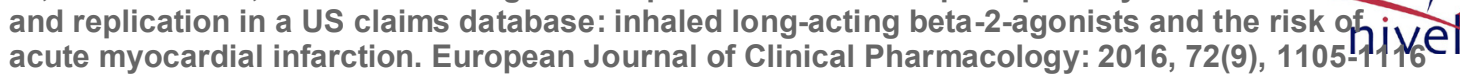

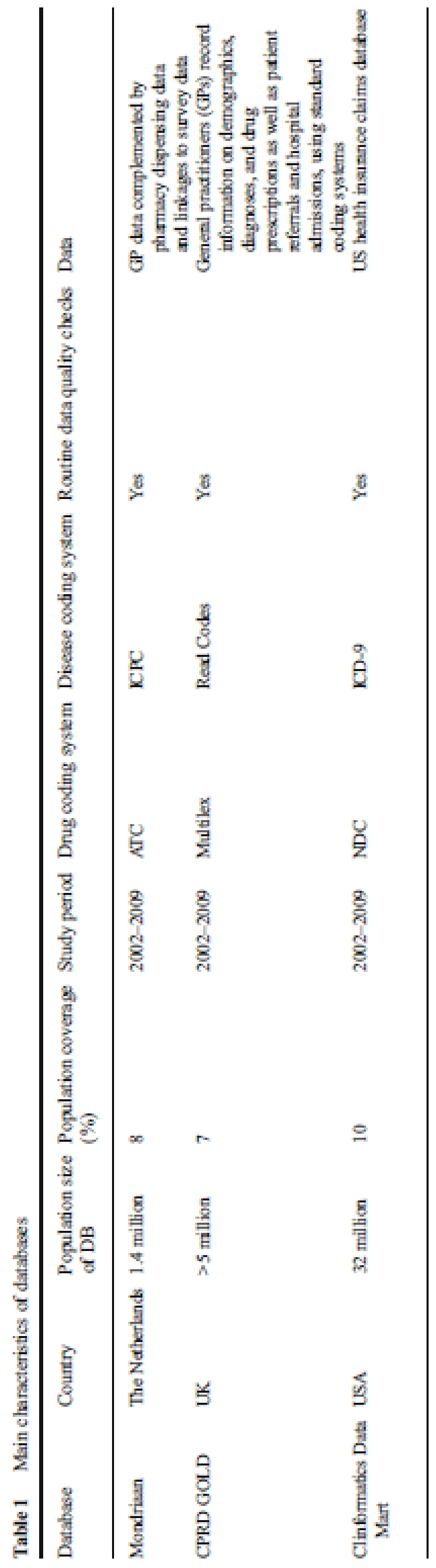


Afonso, A., Schmiedl, S., Becker, C., Tcherny-Lessenot, S., Primatesta, P., Plana, E., Souvereing P., Wang, Y., Korevaar, J.C., Hasford, J., Reynolds, R., Groot, M.C.H. de, Schlienger, R., Klungel, O., Rottenkolber, M. A methodological comparison of two European primary care databases and replication in a US claims database: inhaled long-acting beta-2-agonists and the risk of pive acute myocardial infarction. European Journal of Clinical Pharmacology: 2016, 72(9), 1105-11 16

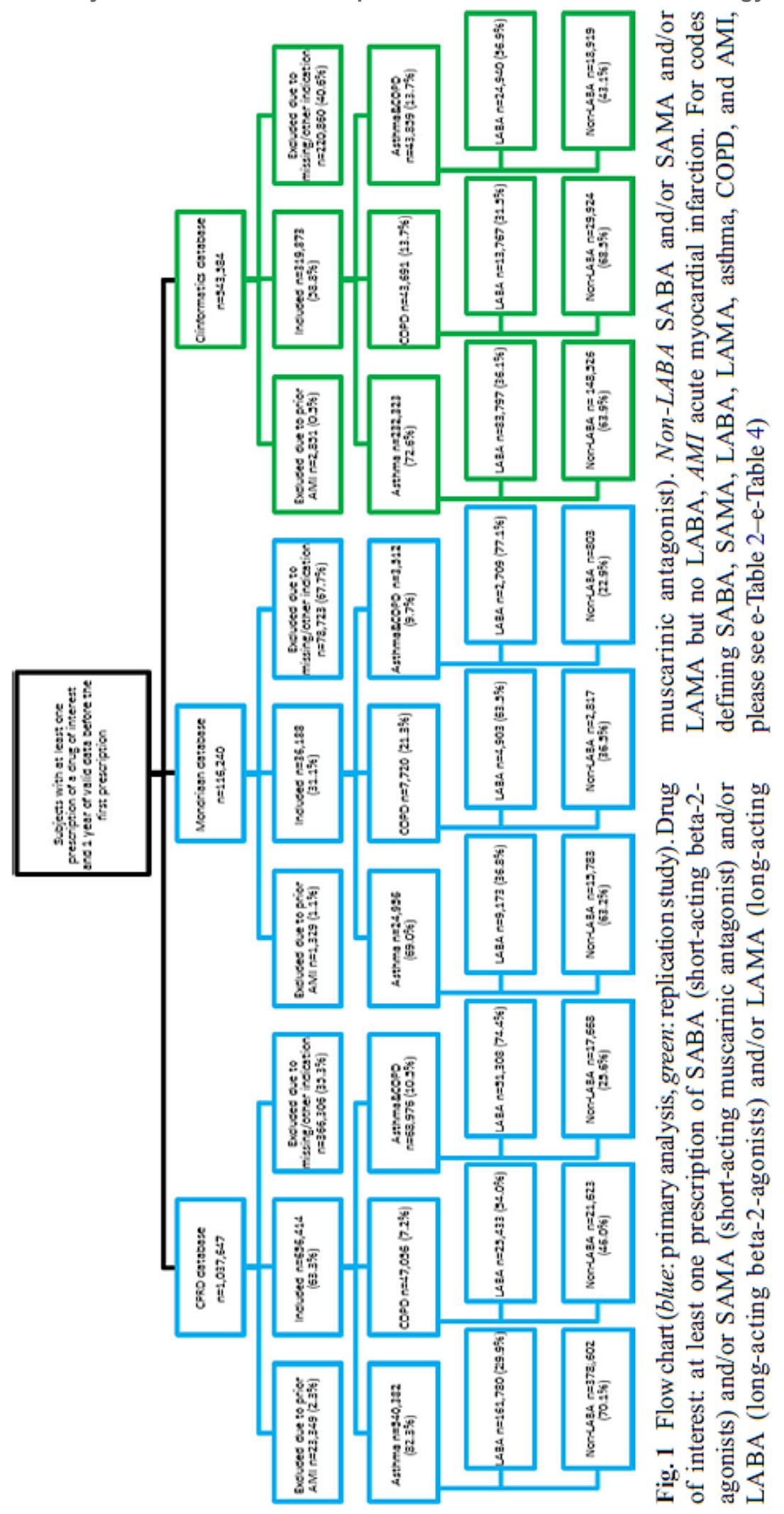


Afonso, A., Schmiedl, S., Becker, C., Tcherny-Lessenot, S., Primatesta, P., Plana, E., Souverein P., Wang, Y., Korevaar, J.C., Hasford, J., Reynolds, R., Groot, M.C.H. de, Schlienger, R., Klungel, O., Rottenkolber, M. A methodological comparison of two European primary care databases 7

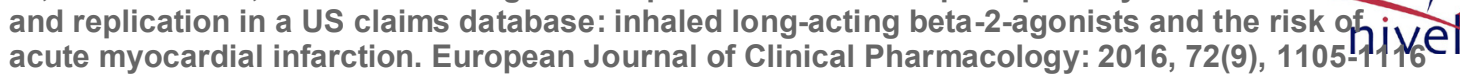

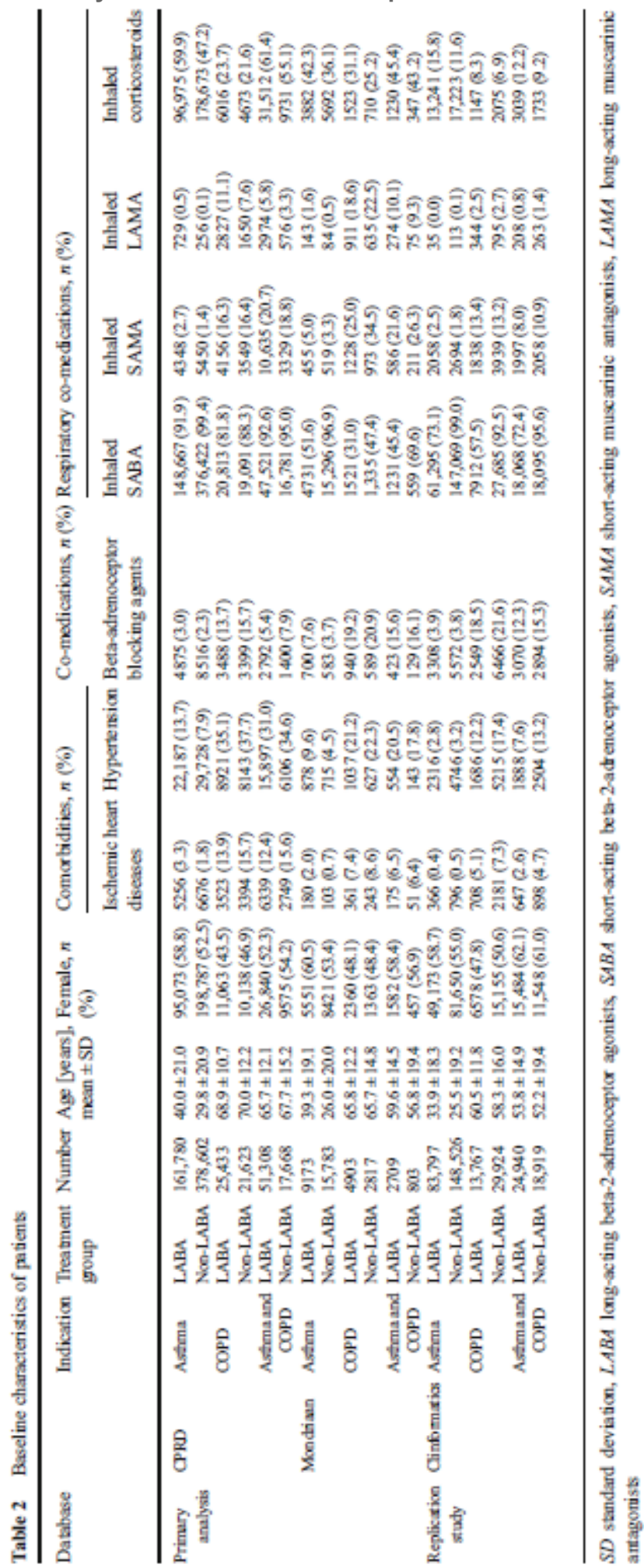


Afonso, A., Schmiedl, S., Becker, C., Tcherny-Lessenot, S., Primatesta, P., Plana, E., Souverein P., Wang, Y., Korevaar, J.C., Hasford, J., Reynolds, R., Groot, M.C.H. de, Schlienger, R., Klungel, O., Rottenkolber, M. A methodological comparison of two European primary care databases 7 and replication in a US claims database: inhaled long-acting beta-2-agonists and the risk of jVe acute myocardial infarction. European Journal of Clinical Pharmacology: 2016, 72(9), 1105-11恹

Asthma

COPD

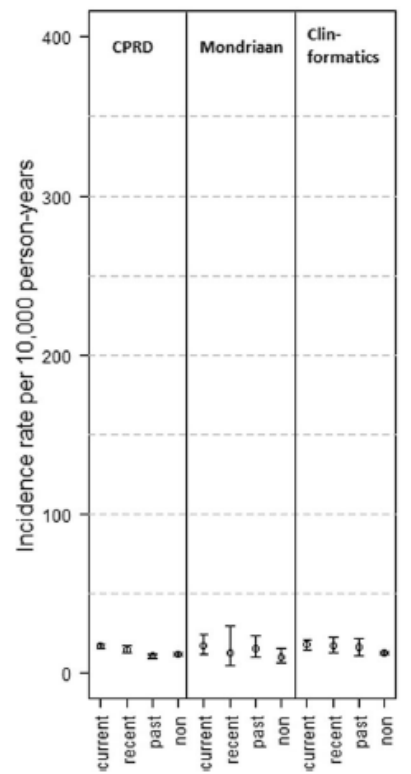

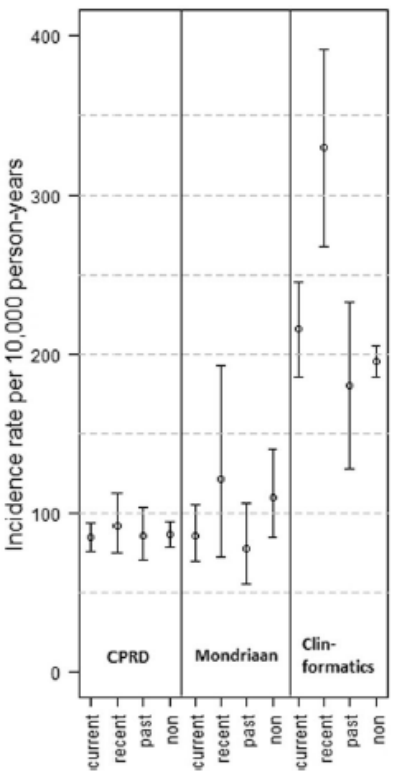

Asthma\&COPD

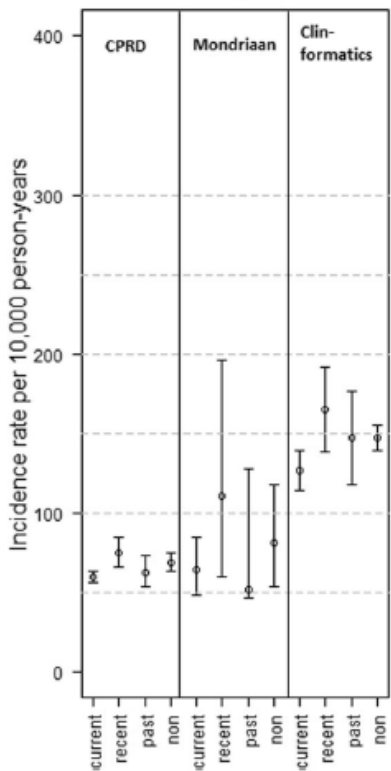

Fig. 2 Crude incidence rates of acute myocardial infarction (AMI-IRs) per 10,000 person-years stratified by indication, database, and exposure period (current current LABA user, recent recent LABA user, past past LABA user, non current non-LABA user)

Asthma - Model1

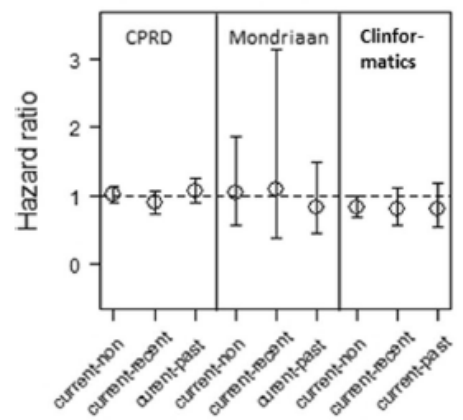

Asthma - Model2

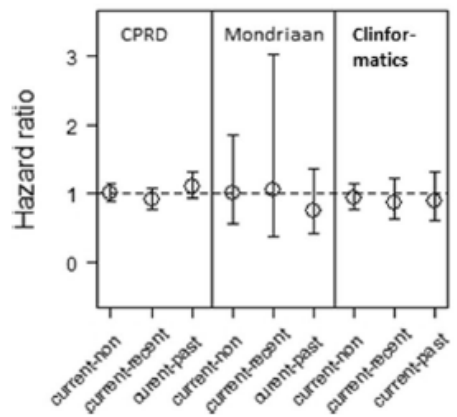

Fig. 3 Adjusted hazard ratio of acute myocardial infarction (AMI-aHR) stratified by indication, database, and comparison group. Model 1 adjusted for sex and age (time-dependent); model 2 adjusted for sex, age (time-dependent), comorbidities (e-Table 5), and co-medications (e-
Asthma\&COPD - Model1
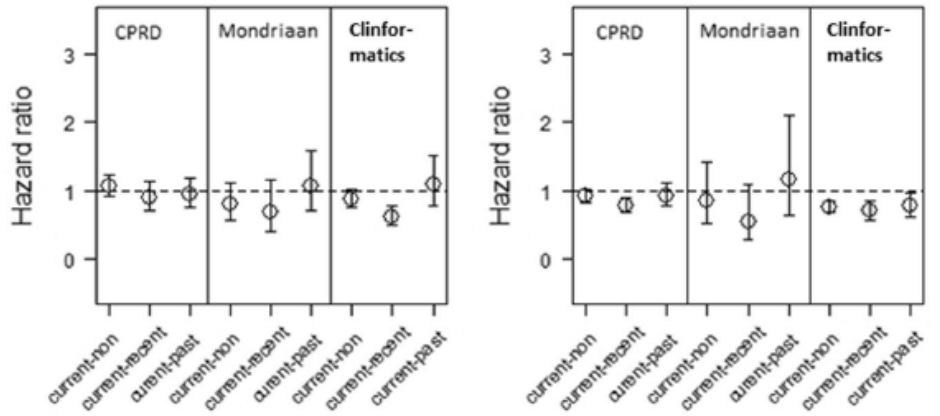

Asthma\&COPD - Model2
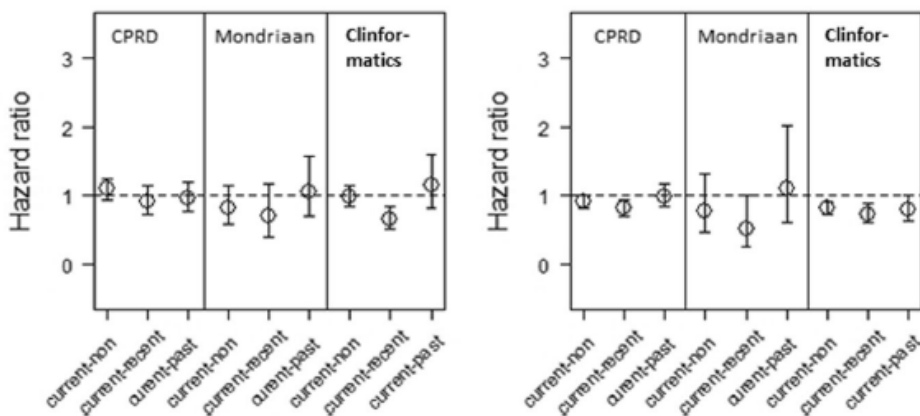

Table 6, e-Table 7). current-non current LABA user vs, current nonLABA user, current-recent current LABA user vs. recent LABA user, current-past current LABA user vs. past LABA user 